\title{
LA ÉTICA CONDENADA: UNA REFLEXIÓN DE LAS CAUSAS Y CONSECUENCIAS DE SU OSTRACISMO EN LA ENSEÑANZA OBLIGATORIA
}

\author{
Enrique Ferrari Nieto \\ Universidad de Extremadura
}

RESUMEN: La inclusión en el curso escolar 2008-2009 de la asignatura "Educación para la ciudadanía" en el currículo de la ESO (con una carga lectiva de una hora semanal) vino acompañada de una polémica desmedida, alentada desde el ámbito político, por el temor a su posible carga dogmática, aunque el contenido ya estaba ahí antes, de modo transversal. Tres años después el debate parece cerrado (por el momento). Con lo que la cuestión ahora, con el tema reposado, podría ser atender a la -de verdad-gran damnificada por esa reforma educativa: la asignatura de Ética, en $4^{\circ}$ ESO, que en muchas comunidades autónomas pasó de dos sesiones a una sola sesión a la semana, con la mitad de su contenido, además, enfocado al civismo.

Mi propuesta es un análisis de las causas y las consecuencias del desprecio a la Ética como materia filosófica en el currículo escolar. Las causas arrancan de la incapacidad general para distinguir la ética de la moral: para entender los distintos niveles (uno filosófico, el otro no) en que se mueve cada una. La consecuencia primera es, con la deslegitimación de la asignatura, la imposibilidad de motivar en el alumno una auténtica reflexión ética.

PALABRAS CLAVE: Filosofía, moral, autonomía moral, adolescencia, vocación.

\section{CONDEMNED ETHICS: A THOUGHT ABOUT THE CAUSES AND CONSEQUENCES OF OSTRACISM IN SECONDARY EDUCATION}

\footnotetext{
ABSTRACT: In the 2008-2009 school year "Education for Citizenship" is incorporated into the curriculum of the ESO (one session per week). Inclusion is very controversial: from the political sphere, it is feared the potential burden dogmatic. After three years, the dispute seems settled. Now the question is to
} 
meet the high damaged by the educational reform: the subject of Ethics ( $4^{\mathrm{o}}$ ESO). In many regions decreased from two sessions to one session a week. With half of its content focused on civic education.

My proposal is an analysis of the causes and consequences of disregard for Ethics (as philosophically) in the school curriculum. The causes stem from the general inability to distinguish the ethical and moral: to understand the different levels (one philosophical, the other not) that moves each. The first consequence is the inability to motivate the student a genuine ethical reflection.

KEYWORDS: Philosophy, categorical imperative, moral, moral autonomy, adolescence, vocation.

Recibido: 16/10/2011

Aceptado: 20/04/2012

\section{LA CONFRONTACIÓN DE TEORÍA Y PRÁCTICA}

A pesar de las discusiones encarnizadas sobre el tema de hace solo dos o tres años, en un trabajo que quiera ser honesto con la experiencia propia no es posible sostener una actitud demasiado tajante, o positiva o negativa, en la presentación y evaluación del peso de la ética en el currículo escolar español. Porque hay siempre una distancia entre el momento de la reflexión, en la escritura, donde el perfil de las ideas lo da uno mismo, y el de su experimentación, en el que ese perfil es el resultado de la confrontación de factores imprevisibles donde la destreza del que escribe necesita sus aliados, no siempre con la mejor disposición. Con ese primer momento, uno solo frente a sus libros y su ordenador, es fácil acabar moderadamente satisfecho, feliz con el resultado. Es una cuestión de horas. Después de todo, es desconcertantemente fácil amoldar las ideas, si no hay referentes fuera, para que se Ileven bien entre ellas: el concepto, con el que en su día habíamos creído poder discernir la realidad, no deja de ser ficción, una creación mental que quiere servir de intermediario entre (todavía con la fórmula idealista) el sujeto y el objeto, aunque a veces se haya querido dejar en suspenso esta obviedad, para no caer en la desolación de movernos solo entre construcciones propias, con menos cimientos de los que quisiéramos. En cambio, con el segundo momento, el práctico, a ese artesano que nos representaba antes, que con sus manos experimentadas había conseguido dar forma al discurso aparente, se le encarga ahora rematar el trabajo con guantes de boxeador. Para que aquí los artificios no sirvan. Con lo que la honestidad del teórico para un trabajo, como digo, de presentación y evaluación de una práctica cualquiera, tiene que ser la atención constante a los arneses de una experiencia en el aula.

Nuestro tiempo no puede considerarse a sí mismo precisamente una atalaya, pero tras las convulsiones del siglo XX -también en los intentos por legitimar una actitud, una posición ante el conocimiento y ante la sociedad- vivimos ahora un periodo más tranquilo, más equilibrado, recogiendo las grandes aportaciones de esas reflexiones pasadas, rescatadas de tanta morralla, en una síntesis que quiere evitar los excesos. En sus últimos espasmos se quedó cerca el relativismo, una herramienta que al principio fue tremendamente útil para enfrentarse a la dictadura de las 
ideas contundentes, que se creían irrebatibles, del racionalismo, pero que luego, en su deriva, Ilegó a ser capaz de justificarlo absolutamente todo con solo construir un contexto con el que explicarse. Queda todavía cierta tendencia (en principio bien intencionada, pero inconsistente) hacia el relativismo (extremo) en diversos grupos sociales, pero no tiene ya una legitimidad como actitud de base, como la predisposición a aceptarlo todo por no poder echar mano de unos principios universales: ese relativismo cultural al que le ha tocado ya tantas veces hacer de abogado del diablo. Pasa el tiempo, y los fervores de hace 30 o 40 años con la posmodernidad, con el fin de los grandes relatos de Lyotard, de cualquier fundamento, se han ido apaciguando, aun reconociendo el valor de su crítica a algunos desmanes de la modernidad, lo suficiente al menos como para volver sin complejos a aquella sentencia de Bernardo de Chartres, en el siglo XII, que constituye la justificación más concentrada de la pedagogía: Somos enanos a hombros de gigantes, dijo el filósofo neoplatónico. Como una actitud saludable que no quiere volver al principio de autoridad incuestionable sino entender, asumir y enriquecerse con la herencia de los grandes pensadores pasados: Podemos ver más, y más lejos que ellos -continúa-, pero no por alguna distinción física nuestra, sino porque somos levantados por su gran altura. Es decir, aprovechándonos de ellos, utilizándolos. Sin una actitud beata, como diría Ortega y Gasset (Ortega, VIII, 352). Como si los grandes autores estuvieran petrificados, inalcanzables en su pedestal. Como si se nos olvidara, por ejemplo, que Nicómaco, el referente del texto más importante de Ética de Aristóteles, que le da el nombre, era su propio hijo. Que siempre entre lector y autor hay un parentesco, aunque sea a la fuerza lejano (Cf. Savater, 33-34).

\section{LOS RIGORES DE UN IMPERATIVO CATEGÓRICO PARA APRENDICES}

No es necesario sacar conclusiones de la coincidencia, pero tanto en filosofía (en términos generales) como en ética (concretamente la filosofía moral) hay dos grandes nombres que son todavía hoy los grandes puntos cardinales. Aristóteles, en el siglo IV a.C., que la creó como disciplina organizada, y Kant, que en el XVIII, tras más de dos mil años, decide acabar con los principios vigentes desde Aristóteles de una ética de los fines, teleológica, para, en sintonía con su sistema filosófico, concebir el bien como un deber ser apriorístico. El bien, dirá, no puede depender de los resultados de la acción, siempre contingentes, sino de criterios absolutos, lo que afianza la ética como disciplina filosófica. Muy someramente: Kant, aunque reconoce que no podemos conocer lo absoluto -tema de la tercera parte de su Crítica de la razón pura-, entiende que sí tenemos un contacto con algo absoluto en el ámbito de la moral, en la conciencia moral, porque al hacer discriminaciones de orden ético son necesarios ciertos principios morales de carácter absoluto. Establece uno: la buena voluntad, absolutamente buena porque así la reconocen todos y porque lo es intrínsecamente, en sí misma, al margen de sus resultados. Pero como el hombre no es totalmente racional, y está condicionado también por lo emocional (en el siglo XVIII peor valorado que ahora), esta buena voluntad, en lugar de realizarse espontáneamente, se convierte a veces en deber: Punto en el que Kant -en su Fundamentación de la metafísica de las costumbres- formula su imperativo categórico (imperativo porque es una exigencia y categórico porque tiene carácter absolu- 
to): Obra de tal forma que la máxima que rija tus actos pueda valer como principio de legislación universal y necesario.

Cualquier imperativo hipotético se fundamenta en fines relativos. Pero el imperativo categórico tiene que fundamentarse en un fin absoluto, que es, más allá de sus fórmulas, el gran legado de Kant: Qué puede considerarse como único fin absoluto. Porque la respuesta -solo son fines absolutos las personas- devuelve al hombre, frente a cualquier tentación utilitarista, a un primer puesto incuestionable. Lo radicalmente inmoral, dirá, es la utilización de cualquier persona como un medio, no como un fin en sí misma. El Kant de la crítica de la razón pura le había tenido que dar la razón a Hume, como en tantas otras cosas, pero desde su razón práctica (lo que constituye su ética) no puede concebir a la persona solo como una suma de fenómenos: la persona -escribirá- tiene una naturaleza en virtud de la cual es un fin en sí mismo. Una lección que, a pesar de haber sido históricamente zarandeada una y otra vez, tiene que ser imperecedera: la respuesta definitiva al Homo homini lupus. Como si Kant hubiera hecho fuerza del lado de Séneca, que escribió aquello de que el hombre es algo sagrado para el hombre, para contrarrestar el apoyo, un poco anterior, de Hobbes a la sentencia potentísima de Plauto.

El problema viene del excesivo formalismo y del excesivo rigor del imperativo categórico: dos críticas que han estado ahí desde el principio, pero que orientado el imperativo a la educación de los jóvenes parecen más infranqueables que nunca. Quizá no tanto para el teórico. Pero sí para el profesor que tiene delante a veinte o treinta chavales para los que el pobre Kant es solo una momia hasta entonces absolutamente alejada de sus vidas, incluso más que otros filósofos menores pero con más presencia en la vida cotidiana que construyen los medios: para el profesor que tiene por delante un trabajo de acortar distancias. Por ejemplo: Kant distingue cuatro tipos de actos morales, que explica desde un mismo caso en el que va cambiando un solo elemento, la persona a salvar: Ante un precipicio, un individuo está a punto de caer, y está en la mano de otro individuo que se salve o no. Las opciones, en principio, son dos: salvarlo o no salvarlo. Pero Kant llega a distinguir hasta cuatro tipos de actos morales: 1) Un acto contrario al deber: cuando no le salva la vida a la persona, por ejemplo porque le debe dinero a la víctima y de este modo no tiene que devolvérselo. 2) Un acto de acuerdo con el deber por inclinación mediata: cuando le salva la vida a la persona, pero porque es esta la que le debe dinero, con lo que solo salvándola lo puede recuperar. 3) Un acto de acuerdo con el deber por inclinación inmediata: cuando le salva la vida a la persona porque, por ejemplo, la ama: quiere salvarla como un fin en sí mismo. Y 4) un acto por deber, como salvar a un enemigo, sin que medien inclinaciones, solo porque es lo indicado por el deber. Con lo que Kant defiende no que tenga que haber un conflicto entre las inclinaciones, sino que, en caso de conflicto, debe primar el deber. El carácter moralmente bueno solo lo da el deber. Por lo que en el segundo y en el tercer caso, en las que son las inclinaciones las que determinan la acción, el acto es moralmente neutro. Que es lo que les cuesta tanto entender a los chavales, porque no pueden separar las consecuencias de la motivación de la acción, donde Kant pone la carga moral, de un modo poco intuitivo, más filosófico. Entienden mejor los ejercicios fundamentados en los principios utilitaristas, midiendo las consecuencias de sus acciones (lo que se les ha pedido hasta entonces). Por ejemplo, el de la balsa a punto de 
naufragar, que necesita liberar peso arrojando al mar a uno de sus pasajeros, que permite un debate más sencillo, más intuitivo, convertido en un juego de estrategia, para elegir al condenado, que depende directamente o bien de su condición de potencial desahuciado (si es anciano o enfermo o suicida) o por la poca rentabilidad que puede sacar de él el mundo (si es un drogadicto o un vago) o por merecérselo más que cualquier otro, en un orden moral poco escrupuloso (si es un asesino o un ladrón), o por peligroso (el más pesado o un caníbal): Un ejercicio más viable y divertido, sin duda, para la clase, porque no necesita por debajo un discurso complejo bien desarrollado en torno a qué es ético que Ilevaría muchas horas; pero que acarrea todos los vicios de la moral utilitarista más ramplona, que se afianza como único recurso en la conciencia de los alumnos. Como si -aquí habla el teórico indignado- el tope que pudiéramos exigirles en su reflexión ética fuera esto, renunciando a cualquier esfuerzo por construir un armazón filosófico sólido para pensar la moral. Pero también como si -aquí habla el profesor impotente- hubiera más posibilidades que esto, con una sola asignatura en los cuatro años de enseñanza secundaria, de una hora o a lo sumo (dependiendo de cada comunidad autónoma) de dos, sin ninguna noción de filosofía previa.

\section{Posibles E IMPOSIBLES}

Como digo, en los currículos hay una sola asignatura de Ética, en $4^{\text { }}$ ESO, para los cuatro años de secundaria y los dos años de bachillerato. En bachillerato, de hecho, no hay nada, aunque en su primer curso sí hay una asignatura de religión (católica) que puede ser sustituida por otras opciones para aquellos alumnos que no deseen cursarla. La carga lectiva de esa asignatura de Ética es de una o, en unas pocas regiones, dos clases semanales: en cualquier caso insuficiente. Antes eran dos horas en todos los currículos, pero hace poco, en el curso 2008-2009, con la implantación de Educación para la ciudadanía en $2^{\circ}$ ESO, algunas comunidades autónomas redujeron de dos a una clase semanal la Ética para hacerle sitio a la nueva asignatura, que tiene también algunas nociones que merodean la ética pero más enfocadas a lo cívico, sin ninguna pretensión filosófica; y la convirtieron, aún más, en un seminario o una charla semanal. La polémica, durísima, vino por otras cuestiones, con la acusación de determinados segmentos de la población de que la nueva asignatura era doctrinaria, pero el daño real fue para la vieja Ética (reconvertida en Ética y Ciudadanía), que al perder horas perdió el rigor de su contenido. El profesor se encontró de pronto con una clase a la semana para fijar tres tipos de contenidos: 1) una base conceptual de la ética, 2) la historia de las reflexiones éticas y 3) un contenido social y cívico adaptado a alumnos de 16 años.

La cuestión es que en los dos primeros bloques de contenido el profesor tiene que partir de cero: los alumnos nunca han estudiado las nociones que les puedan servir para encofrar, aunque sea rudimentariamente, las reflexiones (auténticamente) éticas. Pero desde los altares pedagógicos que iluminaron la proeza de mutilar la asignatura no se la reconoció como inicio del trayecto, sino como continuación de un camino, tedioso por lo largo, que se inicia en los primeros años de escolarización, como materia transversal que atraviesa todas las demás. De ahí su justificación para acortarla, cambiándole el contenido (achicándolo e insistiendo en lo cívico) y 
repartiéndola en dos cursos con la Educación para la ciudadanía. Lo que sugiere que quienes planificaron este último cambio no conocen bien la diferencia radical entre la ética y la moral, como si una y otra fueran lo mismo. Mi impresión es que, centrados en la introducción de una nueva asignatura de valores cívicos, uno de los puntos más visibles para el sistema educativo en el programa electoral del PSOE cuando accedió al Gobierno, no se valoró suficientemente los cambios que había que introducir en la asignatura de Ética para cuadrar horarios y contenidos. Como si su partición y nueva orientación cívica fuera solo un modo de hacerla más eficaz, más útil, evitándole reflexiones metafísicas que, en el mejor de los casos, piensan, no sirven para nada. Porque se usa moral y ética indistintamente, como si fueran sinónimos. También en la prensa. Es un mal muy extendido, también entre políticos y pedagogos, no distinguir una y otra: lo que en cualquier temario debería ser la primera lección: La moral es el conjunto de costumbres de un individuo o una sociedad. La ética -la filosofía moral- es una reflexión racional, crítica, que tiene como objeto la moral. Como la estética tiene como objeto de reflexión el arte, sin confundirse, o la ontología el ser. Por lo que el contenido moral que el alumno ha ido recibiendo desde los primeros cursos, como contenido transversal, como un decálogo de las normas legitimadas por su sociedad, no es el objeto de la asignatura de Ética, sino la materia prima de la reflexión con la que esta constituye un nuevo contenido de segundo nivel. En la moral el contenido es directamente la norma moral. En ética el contenido es la reflexión sobre la norma moral. Con lo que la diferencia entre una y otra materia viene dado por la mediación que supone la reflexión racional: un ejercicio difícil que le exige al alumno una madurez intelectual y una capacidad de abstracción que solo se desarrollan al final de la enseñanza secundaria.

Una vez reconocidos los espacios para la moral y la ética, la segunda lección de la asignatura -que enlaza los puntos 1 y 2 con el 3-debería ser la diferencia entre heteronomía y autonomía moral: los modos de aceptación de las normas morales: con el primero el individuo acepta unas normas que le vienen de fuera, se somete a ellas, y con el segundo establece sus propias normas morales en su proceso de madurez, que refleja de algún modo la actitud desde la moral y desde la ética, como un camino que se abre a lo práctico: en los alumnos con el paso de la obediencia (acrítica) a los valores construidos en su comunidad a la reflexión personal de este bagaje para determinar qué valores hacer suyos (propiamente suyos, recreados por el joven de nuevo) y cuáles desechar, tras un periodo, el de la adolescencia, de negación indiscriminada de los valores heredados para poder dar con su propia personalidad. Pero, a partir de aquí, qué puede hacer el profesor, arrojados los esquemas teóricos al escenario del aula: ¿Centrarse en los contenidos del temario? ¿O intentar hacer reflexionar al alumno sobre la moral, en un ejercicio más práctico? Con una o dos horas semanales no puede abarcarlo todo; pero centrarse en los contenidos no potencia el paso del alumno a la autonomía moral, y dejar a un lado los contenidos y dejar que el alumno reflexione sin una base conceptual sólida es moverse sobre arenas movedizas. En cualquiera de los casos, no se consigue colocar al alumno frente a la cuestión ética como reflexión filosófica, racional y autónoma: muy importante para alejar a los estudiantes de la idea de que la ética es un decálogo de buenas acciones, esa confusión tan común. Aunque luego venga una segunda batalla, todavía más cruda, con el papel que debe jugar la filosofía, con dos 
años en bachillerato pero cada vez con menos horas. Decía Ortega que a la filosofía le viene bien ese espacio minúsculo que la sociedad le ha dado, como disciplina inútil, para poder trabajar tranquilos, sin demasiadas intromisiones (Ortega, IV, 199). Pero la realidad es -sin esa explosión de optimismo- que, consecuente con el valor que la sociedad le da a la filosofía, sin saber muy bien qué es, como denunciaba Lázaro Carreter, la tendencia es a rebajar sus horas en el currículo escolar: También en ética, o diluirla con la moral, procurando que el análisis no despegue demasiado. Lo que a los alumnos les complica también su primer año de bachillerato, porque sin una ética propiamente filosófica se pierde el mejor acceso, la mejor preparación para entrar en la filosofía, al vislumbrar sus contenidos y métodos, pero también al potenciar su incipiente capacidad de abstracción.

La ética es filosofía práctica, una filosofía que se realiza, que se concreta en la realidad, como una reflexión continua con la que el individuo va dando las distintas soluciones a las opciones o a los requerimientos que se plantean en su vida. Pero para cada capítulo de la biografía de cada uno las respuestas no están en un listado definitivo de máximas, no basta con la memoria para recordar la cita exacta que se adecue mejor, como hacen los predicadores de las películas, sino que hay que construirlas en cada momento: cada acción exige su reacción: una respuesta creada ex profeso, creada (o actualizada) por el individuo que se enfrenta a la circunstancia, porque, como actor, su papel no se lo ha escrito otro, sino que lo ha escrito él mismo, con sus fuentes y sus deudas, pero con un último repaso suyo. Para el que el imperativo categórico -es verdad- se vuelve casi imposible como principio cotidiano. Demasiado rígido y formalista. Pero en nuestro tiempo hay otras éticas más realizables, más cercanas a nuestra sensibilidad, a los temas que nos son más actuales, como el medio ambiente, la bioética o el multiculturalismo, que pueden orientar y ayudar a conformar una ética propia con ciertas garantías. Pero para llegar aquí es forzosamente necesario (al menos es el camino más corto) que el individuo, en su etapa escolar, haya tenido un profesor que se haya detenido, en sus clases de Ética, en las propuestas anteriores, en esa trayectoria de 2500 años, porque llegar a ellos directamente es dejarlos suspendidos en el vacío, cortado el diálogo histórico entre las distintas propuestas éticas. Es una pena, por ejemplo, no llegar a explicarles a los alumnos la ética -que nos es tan cercana- de Ortega y Gasset, impregnada de Aristóteles, Nietzsche y otros, de la vida entendida como proyecto: En un momento en que los alumnos tienen que decidir por primera vez qué quieren hacer en sus vidas (en los estudios), podría venirles muy bien esa comprensión de cada uno de ellos como un arquero que lanza una flecha: la vida que se hace cada uno, sin mayores determinismos. Porque, decía Ortega, la vida es una tarea: cada uno es un novelista que se escribe su propia vida, aunque en el marco de una circunstancia, de un entorno. La vida es lucha con el entorno: un dinamismo dramático entre yo y el mundo, escribe Ortega, en el que solo vive de verdad el que vive su vocación. La mayoría de los hombres no atienden su llamada; y falsifican su vida, se estafan a sí mismos con una falsa trayectoria vital. Menos los héroes, como Don Quijote, porque lo heroico es un acto real de voluntad: es ser lo que no se es aún. Un héroe es el que, con ademán deportivo, se resiste al hábito. El que abandona las costumbres y herencias en su deseo por innovar la vida. Porque la vocación -la define- es un programa íntegro e individual de existencia. Con un sentido más amplio que el 
común. Sin limitarlo a la profesión, que es algo genérico. Porque la vocación, como proyecto vital, para ser auténticamente, dice, es algo singularísimo, ultraconcreto, como la persona. Con el estímulo de Píndaro (filtrado por Nietzsche) para una propuesta moral desde la razón vital: una llamada a la vocación, a seguir cada uno su auténtico destino (cada hombre, dice, es constitutivamente moral porque tiene que hacer su vida y es responsable de ella), que adopta el imperativo del griego: "Ilega a ser el que eres", el único aceptable frente a otras imposiciones de éticas mágicas: "Esa íntima conciencia constantemente nos dice quién es ese que tenemos que ser, esa persona o personaje que tenemos que esforzarnos en realizar, [...] una voz que es Ilamada hacia nuestro más auténtico destino; en suma, la voz de la vocación, de la personal vocación" (Ortega, IX, 513-514). Una ontología que continúa Marías (tan olvidado hoy) desde el "yo soy yo y mi circunstancia" de Ortega, como un comentario perspicaz con el que establece una antropología metafísica del hombre como ser futurizo. El hombre, como novelista que es de sí mismo para Ortega, debe decidir qué posibilidades realiza. Con lo que Marías propone una antropología que define al hombre como un ser que, como había dicho antes su maestro, no es, evidentemente, futuro, pero sí futurizo, porque es su previsión del futuro lo que determina su presente. Un privilegio ontológico exclusivo del hombre: ningún animal piensa en su futuro. Soy presente, dice Marías, pero orientado al futuro, vuelto a él, proyectado hacia él. El futuro o la posibilidad no existe, actualmente, en mi presente, pero son mis proyectos futuros los que confieren su carácter de mundo al mundo material y presente. Es decir, lo que constituye a la persona es su carácter programático, porque la persona, escribe Marías, no está ahí, sino que está viniendo, indigente, menesterosa, por la irrealidad de la anticipación. Como un proyecto siempre inconcluso. No hay identidad en una persona. Hay mismidad: soy lo mismo, pero no el mismo, porque el yo pasado no es el yo, sino solo una circunstancia que se encuentra el yo presente. Con lo que la vida es, al final, la organización interpretativa o teórica; y, la realidad, el escenario de mi vida: Mi vida -dice- es la organización real de la realidad. Es buscar un sentido, una interpretación de sí misma. Cuya forma empírica es la instalación, en último término como ser corpóreo, encarnado, como decía Marcel, en el mundo.

Pero para llegar aquí (o a los procedimentalistas, o a la ética de mínimos o al debate en torno al Proyecto Gran Simio o el genoma humano) y poder reflexionar con calma sobre la cuestión hace falta tiempo. La filosofía, la reflexión, exige mucho tiempo. Decía Josep Pla que tener siempre prisa es la excusa para no hacer nunca nada (Pla, 111). Otra cosa es exponer unas cuantas normas y unos cuantos lugares comunes. Por lo que la cuestión en último término es determinar hasta qué punto se puede vaciar de filosofía las asignaturas de ética (directamente proporcional al tiempo dedicado) sin hacerles perder su sustrato, algo inevitable con una clase semanal. Hay dos puntos complicados: 1) la dificultad de los alumnos para asimilar la abstracción que requiere la filosofía y 2) las voces que claman contra la filosofía como disciplina y asignatura poco útil, como si fuera una especie de retórica prescindible. Que han alimentado la tentación de convertir la Ética de 4 ESO en una maría, en una asignatura de chichinabo, de relleno: en discusiones pueriles que pueden ser interesantes en tanto que son temas que afectan a los jóvenes, pero sin ningún contenido sólido, o en moralina, o en un desesperado intento del profesor al tratar de meter un contenido serio en 
una clase semanal (45 o 50 minutos) que el alumno recibe como solo teoría, con el temario blindado, no como punto de partida para la reflexión.

\section{LoS PAPELES DE MORAL Y ÉtiCA PARA los ADOLESCENTES}

Pero decía al principio que no es posible sostener una actitud demasiado tajante cuando se le pide a la exposición una confrontación con la realidad, con su concreción en el aula. Desde el lado de la teoría es fácil posicionarse desde una actitud determinada, pero del lado de la práctica esa actitud es, siendo más sincera, también más voluble. Lo que, con sus claras desventajas, se permite también el atisbo de un optimismo menos razonado, sin la necesidad de mantenerlo a lo largo del tiempo, frente a la maraña de pesimismos y nostalgias que constituyen la conciencia colectiva sobre la pedagogía actual, a remolque, después de todo, de la conciencia colectiva sobre nuestra sociedad (pos)moderna y nuestra juventud.

El debate que trajo la asignatura de Educación para la ciudadanía no se cerró, o cerró en falso, con su implantación en el currículo escolar pero con muchas voces disconformes: un síntoma de las dudas en torno al conjunto de valores que buscan y necesitan nuestras comunidades. Los sólidos principios morales que podían con todo han dejado -para muchos teóricos muy leídos, como Bauman o Lipovetsky- un hueco enorme que en una fase posmoralista, como la nuestra, no hay prisa por rellenar, al menos con el conglomerado anterior, que focalizan con el ideal de la abnegación. Desde ese punto de partida pesimista, como el de Bauman en Comunidad, algo que la comunidad estética no hace en modo alguno es tejer entre sus participantes una red de responsabilidades éticas, y por tanto de compromisos a largo plazo (Bauman, 86). Pero posmoralista, dice, para remontar el vuelo, no significa posmoral. Con el historicismo aprendimos que una moral universal es un engaño porque el hombre, el sujeto moral, no puede trascender su contexto. No sería posible, entonces, diseñar grandes imperativos éticos; o, al menos, no sería urgente, pero -bajados al terreno de la práctica- sí habría que establecer unos principios básicos de convivencia en un proceso democrático, llegar a un consenso humilde pero eficaz que proporcione el marco adecuado de actuación en las relaciones interpersonales. Lo dijo Freud: en lo social, la moral es el resultado de una adaptación funcional de los individuos. Una convivencia a la que se accede, en su plenitud, con el cambio de roles que supone el paso de la adolescencia a la adultez. Y los riesgos del tránsito pueden ser minimizados en un diálogo bidireccional entre generaciones, en el respeto a la experiencia y el reconocimiento a nuevas inquietudes. Los juicios morales son prescriptivos, pero deben basarse en razones. Nuestra sociedad no admite -no lo debe hacer- imposiciones morales. Uno de los principios básicos de nuestro tiempo es -aun con sus atropellos- el respeto a la autonomía de los individuos, a su libertad. Y el resultado de ese diálogo tiene que ser la asunción personal de la responsabilidad: el individualismo responsable -la tesis primera en los libros de Lipovetsky - que atiende también a una responsabilidad colectiva cuando la circunstancia exige una toma de decisión común. No hay otro fundamento de obligación que la asimilación racional de unas normas surgidas del diálogo.

La evolución moral, como señaló Piaget en El juicio moral en el niño, sigue un trazado acorde a la maduración psicológica. Como Durkheim, Piaget creía que la 
moral es un sistema de normas, que la esencia de esta es el respeto de los individuos a las reglas. Pero ese respeto puede tener diferentes fases, desde la coacción a la cooperación. Habla de una regla motriz, de valor indicativo: una costumbre; una regla coercitiva, que el sujeto interpreta como algo exterior a él; y una regla racional, nacida del consenso, del acuerdo mutuo, que puede modificarse y que es respetada por ser fruto de un acuerdo. Solo en este último caso el adolescente o el joven puede tomar como propia una ley. Los expertos coinciden en que uno de los problemas de la educación es la negativa de los padres a delegar responsabilidades a los hijos. Se sabe que con una formación adecuada, un chaval de 15 años puede participar en un diálogo que establezca unas normas mínimas de responsabilidad para la convivencia si se le reconoce como agente y se argumenta cada una de las propuestas. Han quedado atrás los monólogos en moral. Es la ética discursiva de Apel y Habermas: el sujeto moral kantiano es sustituido por un diálogo entre participantes de un debate argumental sobre cuestiones prácticas. No consiste en hacer un decálogo de buenas intenciones. Lo necesario ahora es el diálogo fluido intergeneracional en una sociedad compleja que no admite un binomio de prohibiciones y obligaciones. Y la educación, que va más allá del sistema escolar, será quien tenga que abanderar las propuestas, porque es garantía de libertad y de bienestar, porque facilita las relaciones sociales, construye solidaridad y da legitimidad moral a la participación política. Como dijo el Juan de Mairena de Machado: "Nosotros no pretenderíamos nunca educar a las masas. A las masas que las parta un rayo. Nos dirigimos al hombre, que es lo único que nos interesa" (Machado, 164).

El aprendizaje del autocontrol es un universal humano, los cimientos de la convivencia. Se convierte en el hombre en un acto reflejo necesario: para los individuos, que se integran así en su entorno, y para las sociedades, consolidadas en resistencias como esta. Pero un número significativo de personas son excluidas sistemáticamente en los vínculos sociales. Se las desprecia por su posición social, y se las abandona a su suerte. Son, en muchos casos, jóvenes de vida monótona, sin ninguna perspectiva, que saben que pertenecen a esa sociedad, pero le dan la espalda porque se sienten rechazados. Jóvenes en quienes se trunca su proceso de madurez intelectual y emotiva. Son casos extremos. O extremísimos, como el de Alex, el joven protagonista de La naranja mecánica de Anthony Burgess. Pero la adolescencia, una época conflictiva en la construcción de una nueva identidad, lleva en sus entrañas un riesgo potencial, por la falta de mecanismos culturales de contención, de habilidades emocionales y sociales. Las escenas, en la película de Kubrick, son sobrecogedoras: Alex desinhibido, golpeando a un vagabundo o violando a una mujer, y Beethoven de fondo, muy alto. La música del compositor romántico es la incapacidad de control, la confusión, el rechazo de límites, la exageración; la expresión embriagadora de los sentimientos primarios liberados. Pero son la adolescencia y la juventud las que marcan la actitud vital del adulto. Comas distingue tres fases en este periodo: una adolescencia contenida por el control familiar, una juventud transgresora y, por último, un comportamiento más responsable como acceso al mundo adulto. Es un tiempo de cambio y de búsqueda, de acomodación y de perspectivas, en un diálogo con el entorno. Como decía Jean Piaget, la vida afectiva de la adolescencia se afirma por la doble conquista de la personalidad y su inserción en la sociedad adulta. Que es la clave: los jóvenes hoy tienen vedada la entrada a una esfera cada vez más impermea- 
ble. Lo que decide la incorporación de estos a la vida adulta es el trabajo, y el mercado es incapaz hoy por hoy de absorber en condiciones dignas a nuevos miembros. Y ellos lo saben, y se manejan con esas expectativas tan negras ya en el último curso de la enseñanza obligatoria. Se extiende el síndrome de Peter Pan, el miedo a crecer: no son capaces de asumir hasta muy tarde las responsabilidades que se suponen de la vida adulta. Ruiz de Olabuénaga los Ilama libertos (Cf. Ruiz Olabuenaga). Al menos a un porcentaje significativo de ellos. No son ciudadanos libres, sino individuos preadultos, con todos los derechos ciudadanos, libres en su cultura, ética y política, pero esclavizados en su futuro inmediato (un año después, tres años después, ocho años después) al paro y la ayuda familiar.

Pero no sirve de nada ser pesimista. Son muchas, como siempre, las aspiraciones y las frustraciones. La juventud actual -con sus excepciones, evidentemente- no está desesperada ni perdida. Tiene definido su sistema de valores, no tan diferente al de sus antecesores, como va constatando periódicamente el Injuve: un buen sueldo, el éxito en el trabajo, la capacitación cultural y profesional, la familia, etc. Es solidaria. Y confía, dentro de lo que cabe, en los fundamentos del orden social (algunos, de hecho, la acusan de confiada y conformista, a pesar de determinadas manifestaciones como el movimiento 15-M). Y trabaja con la esperanza de ser admitida en ese orden con su nuevo status de adulto, con un papel distinto. Pero los tonos grises de su futuro pueden acabar con sus grandes proyectos, con su entusiasmo. Es la resignación que ha hundido al joven que imagina Welsh, en Trainspotting, en la estación de Edimburgo viendo salir y entrar trenes durante horas: "La vida es aburrida y fútil. Empezamos con grandes esperanzas y después nos acojonamos. [...] Básicamente, vivimos una vida corta y decepcionante; y a continuación morimos. Llenamos nuestras vidas de mierda, de cosas como carras y relaciones para convencernos a nosotros mismos de que no carece todo de sentido" (Welsh, 56). Quien en una estación, lejos, en un banco, se queda absorto durante horas viendo el ajetreo de miles de pasajeros y divaga con torpeza es aquel que cree rotos los vínculos con su comunidad. Quien se sabe aún miembro de esta, pero sin un lugar definido, marginado, porque la vida se orienta en la integración, en la inserción social: el pilar en que se apoyan los valores sociales de los jóvenes es el reconocimiento social, la relación con los demás individuos, que determina la formación de la identidad. Sólo un dios o una bestia puede vivir al margen de la sociedad, decía Aristóteles. Somos animales políticos.

Para los teóricos más negativos se me ocurre la escena de La Balsa de la Medusa, enorme, sobrecogedora, que pintó Théodore Géricault en 1819: unos cuantos hombres desnudos sobre unas tablas improvisadas en medio del oleaje y la tormenta, algunos desfallecidos o muertos, perdidos, desesperados, con los rostros marcados por una tragedia de ocres oscuros. El instante en que lo detiene Géricault es el del avistamiento de una fragata de la marina francesa, que no los recogió. Son los náufragos, símbolo de un tiempo que no cree ya en referentes heredados: el fin de la modernidad: la imposibilidad de remitir a un primer gran relato en la legitimación de las reglas de juego. Quiebra el idealismo y queda el individuo solo. Es Narciso, el paradigma de Lipovetsky: el hombre fragilizado, desestabilizado, porque tiene que construirse completamente solo, sin el apoyo de los marcos colectivos y las normas sociales: el joven que ha crecido sin un principio de autoridad, porque el discurso del maestro ha sido desacralizado y no vale más que el de cualquier charlatán 
que sale por la televisión. Todo es relativo, y no hay de dónde asirse. El individualismo democrático que fue, durante un tiempo, motivo de euforia, es ahora la dificultad de vivir, la inseguridad. Narciso es el actor libre, liberado de imposiciones colectivas, surgido de la deserción generalizada de los valores sociales en el proceso de personalización.

Pero, con el mismo escenario, frente a esta convicción pesimista de asistir a la etapa crepuscular con la que acaba el sistema de valores canónico, enmarcada en el relativismo más visceral, más destructivo, cabe también una actitud menos predispuesta a dramatismos. El adolescente, para fijar su personalidad, necesita reaccionar (un modo de tomar conciencia) contra los valores recibidos. Entonces, desde la moral, es inevitable el conflicto con los valores que ha ido recibiendo en su etapa escolar si quiere distinguirse como sujeto moral autónomo, con una personalidad construida por él mismo, dirigida por él mismo, aunque constituida también (pero no subordinada) por las herencias. Probablemente fracase, porque el alumno va a estar predispuesto a rechazar tantas consignas impuestas. Pero desde la ética, en cambio, la discusión de esos valores (y cuanto más los discuta mejor) es precisamente el estímulo para ayudar al alumno a hacer de este enfrentamiento un acto de libertad y de reflexión racional que posibilite su conversión en un sujeto moral autónomo. Todos esos temores catastrofistas serían, por tanto, solo el mejor contenido, el sustrato, que necesita la Ética -la asignatura- para sus discusiones, como una primera fase para dar no con beatos, sino con sujetos verdaderamente autónomos, de verdad novelistas de sus propias vidas. La pena es que, precisamente ahora, cuando más se requiere, no haya tiempo para fundamentarlo y discutirlo todo.

\section{REFERENCIAS BIBLIOGRÁFICAS}

BAUMAN, Z. (2003). Comunidad. En busca de seguridad en un mundo hostil. Madrid: Siglo Veintiuno.

MACHADO, A. (1984). Juan de Mairena. Madrid: Espasa-Calpe.

ORTEGA y GASSET, J. (1983). Obras completas. Madrid: Espasa-Calpe.

PLA, J. (2010). Viaje en autobús. Barcelona: Destino.

RUIZ OLABUENAGA, I. (1998). La juventud liberta: géneros y estilos de vida en la juventud urbana española. Bilbao: Fundación BBV.

SAVATER, F. (2002). El valor de educar. Barcelona: Ariel.

WELSH, I. (2005). Trainspotting. Barcelona: Anagrama. 